\title{
Pneumocystis carinii
}

National Cancer Institute

\section{Source}

National Cancer Institute. Pneumocystis carinii. NCI Thesaurus. Code C127308.

A species of yeast-like fungi in the family Pneumocystidaceae. P. carinii typically infects the lungs of rats. 